\section{A Historical Reference for Snowboarding Injuries}

\section{To the Editor:}

I have read the excellent article by Dr Christopher Van Tilburg, "Backcountry Snowboarding: Medical and Safety Aspects," in Wilderness and Environmental Medicine (2000;11:102-108) with great interest and appreciation. It sought to include a historical overview of snowboarding and succeeded in large degree. But in this altogether excellent historical recitation, I notice a possible void: the initial snowboarding article. Perhaps, because of the alarming implications contained therein, you might be interested in the details of this pioneering medical article.

I refer to our report in Clinical Sports Medicine (1989; 1:45-55), Chapman and Hall Ltd (London): "First Report of Snowboard Neurological Injury in Winter Sports." It presented the first medical report on snowboarding (and snowboarding head/spine injuries) to be published worldwide. One case is of such severity and near mortality that I believe it could be worth describing in limited detail:

A 15-year-old male high school student was snowboarding off a nearby aerial jump, attempting to negotiate a "tail-grab" or "mule-kick" maneuver. He landed incorrectly and struck his midoccipital area with his own snowboard's tail, losing consciousness. The curved tail of the snowboard acted as a broad spade, impaling the occiput. An emergency physician closed what appeared to be a simple scalp laceration, and the victim was discharged home. At home, the patient developed sudden vomiting, which prompted his mother to consult her home remedy book; she followed its suggestion, "return to the emergency room."

Reexamination revealed stupor, intermittent vomiting, and blurred optic disks. Radiographic studies were then done, and they suggested a penetrating skull and brain injury with laceration of the terminal portion of the sagittal sinus above the torcula.

Emergency surgery required a bilateral occipital approach for repair of the sagittal sinus' terminal segment. An initial trial of 1-mm elevation of the depressed midline occipital bone precipitated aggressive circumferential venous blood flow from the entire $360^{\circ}$. On witnessing this, all available hospital match blood was brought to the operating room before the next step.

After an encircling bone incision isolated the bone of the compound fracture, an assembly of Gelfoam, Surgicell pack, and cotton patties was quickly substituted for the mobilized depressed bone area. During this $<1$ second switch of bone to the tamponage dressing, two 6- to 8-inch continuous spurting vertical columns of ve- nous blood were produced. Massive blood loss for 1 minute produced hemodynamic instability. The sinus repair with dural substitute over the occlusive tamponading dressing at the torcula proceeded concurrent with replacement of 4 units of blood to restore acceptable blood pressure. The victim was equipped with a temporary helmet and was advised to sleep prone. Two months after discharge, he was admitted for cranioplastic repair of the vulnerable midline occipital defect. The next year, he returned to snowboarding ... with a helmet.

The report also included 1) the first reported snowboard-related spinal case, that of a 14-year-old snowboarding aerialist who sustained fracture subluxation of C4-C5 and C5-C6 with transient quadriplegia, and 2) the first case of generalized traumatic seizures precipitated by snowboarding impact.

Earlier citations such as this do not always lend themselves to detection by the current robust and efficient computer searches. This historical omission (unintentional, I'm sure) in no way diminishes the value of the very valuable (and in my opinion, essential) clinical contribution reported in your journal.

John B. Harris, MD, FACS Tahoe Neurosurgical Ski Foundation Pebble Beach, CA, USA 